COMMUNICATIONS IN

ANALYSIS AND GEOMETRY

Volume 8, Number 2, 375-391, 2000

\title{
Quasi-convergence of the Ricci flow
}

\section{DAN KNOPF}

\begin{abstract}
We study a collection of Riemannian metrics which collapse under the Ricci flow, and show that the quasi-convergence equivalence class of an arbitrary metric in this collection contains a 1-parameter family of locally homogeneous metrics.
\end{abstract}

\section{Introduction and statement of main theorem.}

In [1], Hamilton and Isenberg studied the Ricci flow of a family of solvgeometry metrics on twisted torus bundles. This family contains no Einstein metrics, so the (normalized) Ricci flow cannot converge. Hamilton-Isenberg introduced the concept of quasi-convergence to describe its behavior, writing

"...the Ricci flow of all metrics in this family asymptotically approaches the flow of a sub-family of locally homogeneous metrics..."

The intent of this paper is to make that statement more precise. In so doing, we answer a question of Hamilton, who asked whether an arbitrary metric in this class would converge to a unique locally homogeneous limit or would exhibit a more nuanced behavior.

Definition 1.1. If $g, h$ are evolving Riemannian metrics on a manifold $\mathcal{M}^{n}$, we say $g$ quasi-converges to $h$ if for any $\varepsilon>0$ there is a time $t_{\varepsilon}$ such that

$$
\sup _{\mathcal{M}^{n} \times\left[t_{\varepsilon}, \infty\right)}|g-h|_{h}<\varepsilon .
$$

Quasi-convergence is an equivalence relation. Indeed, the standard fact that $|U(V, V)| \leq|U|_{h}|V|_{h}^{2}$ for any symmetric 2-tensor $U$ and vector field $V$ implies that $g$ quasi-converges to $h$ if and only if for all $t \geq t_{\varepsilon}$,

$$
(1-\varepsilon) h(V, V) \leq g(V, V) \leq(1+\varepsilon) h(V, V) .
$$

We now state our result, using notation defined in [1] and to be reviewed in $\S 2$ below. 
Theorem 1.2. If $g$ is any solv-Gowdy metric on a twisted torus bundle $M_{\Lambda}^{3}$, there is a locally homogeneous metric $h$ in its quasi-convergence equivalence class $[g]$. Moreover, if $h$ corresponds to the data $(\alpha(\theta), \Omega, F)$, the locally homogeneous metrics in $[g]$ are exactly those with the data $(\ell+\alpha(\theta), \Omega, F), \ell \in \mathbb{R}$.

Remark 1.3. Similar quasi-convergence of the Ricci flow to a 1-parameter family was conjectured for a class of $\mathcal{T}^{3}$ metrics studied in [2].

The paper is organized as follows. $\S 2$ describes the bundles $\mathcal{T}^{2} \rightarrow \mathcal{M}_{\Lambda}^{3} \rightarrow$ $\mathcal{S}^{1}$ and the solv-Gowdy metrics under study. It turns out that at large times, an arbitrary solv-Gowdy metric $g$ behaves much like locally homogeneous metrics. $\S 3$ quantifies this observation and explicitly constructs a family $h_{\varepsilon}$ of locally homogeneous metrics existing for all $t \geq 0$ which approximate $g$ for times $t \geq t_{\varepsilon}$. In $\S 4$, we show that this family enjoys a certain compactness property which allows us to prove the existence part of the main theorem. The heuristic here is that $g$ resembles a single locally homogeneous metric closely enough that the metrics $h_{\varepsilon}$ are not too far apart at $t=0$. $\quad \S 5$ completes the main theorem by explaining the very special sort of nonuniqueness which can occur: distinct locally homogeneous metrics define distinct equivalence classes unless they differ only by a dilation of the base circle.

Acknowledgement. I wish to thank Richard Hamilton for his helpful. and encouraging comments.

\section{Review of solv-Gowdy geometries.}

We begin by briefly recalling some notation and results of [1]. Readers familiar with that paper may skip this section.

To construct an arbitrary solv-Gowdy metric $g$, take $\Lambda \in \mathrm{SL}(2, \mathbb{Z})$ with eigenvalues $\lambda_{+}>1>\lambda_{-}$. In coordinates $\theta, x, y$ on $\mathbb{R}^{3}$, chosen so that the $x, y$ axes coincide with the eigenvectors of $\Lambda$, define

$$
g \doteqdot e^{2 A} d \theta \otimes d \theta+e^{F+W} d x \otimes d x+e^{F-W} d y \otimes d y,
$$

where $F$ is constant and $A, W$ depend only on $\theta$. Clearly, $g$ descends to a metric on the product of the line and the torus $\mathcal{T}^{2}$. Let $\Lambda$ act on $\mathbb{R} \times \mathcal{T}^{2}$ by $(\theta, x, y) \mapsto\left(\theta+2 \pi, \lambda_{-} x, \lambda_{+} y\right)$. If

$$
A(\theta+2 \pi)=A(\theta)
$$


and

$$
W(\theta+2 \pi)=W(\theta)+2 \log \lambda_{+}
$$

then $\Lambda$ is an isometry, and $g$ becomes a well defined metric on the mapping torus $\mathcal{M}_{\Lambda}^{3}$, regarded as a twisted $\mathcal{T}^{2}$ bundle over $\mathcal{S}^{1}$. Notice that $A$ governs the length of the base circle, while $F$ and $W$ respectively describe the scale and skew of the fibers. We denote arc length by

$$
s(\theta) \doteqdot \int_{0}^{\theta} e^{A(u)} d u
$$

and set

$$
Z \doteqdot \frac{\partial}{\partial s} W
$$

Then we can write the Ricci tensor as

(2.6) $\mathrm{Rc}=-\frac{1}{2} e^{2 A} Z^{2} d \theta \otimes d \theta-\frac{1}{2} e^{F+W} \frac{\partial Z}{\partial s} d x \otimes d x+\frac{1}{2} e^{F-W} \frac{\partial Z}{\partial s} d y \otimes d y$.

The locally homogeneous solv-Gowdy metrics are easily characterized.

Lemma 2.1. A solv-Gowdy metric $g$ is locally homogeneous if and only if $W$ depends linearly on arc length.

Proof. If $g$ is locally homogeneous, then $R=-\frac{1}{2} Z^{2}$ is constant in space. Since $Z$ is continuous, it follows that $\partial^{2} W / \partial s^{2}=0$.

If $Z$ is constant in space, let $P_{0}=\left(\theta_{0}, x_{0}, y_{0}\right), P_{1}=\left(\theta_{1}, x_{1}, y_{1}\right)$ be points in $\mathcal{M}_{\Lambda}^{3}$. It will suffice to construct a diffeomorphism $\Phi: \mathcal{U}_{0} \rightarrow \mathcal{U}_{1}$, where $\mathcal{U}_{0}, \mathcal{U}_{1}$ are neighborhoods of $P_{0}, P_{1}$ respectively, such that $\Phi\left(P_{0}\right)=P_{1}$ and $\Phi^{*} g=g$. If $\Phi$ is given in coordinates $(\theta, x, y)$ by

$$
\Phi(\theta, x, y)=(\tau(\theta, x, y), \xi(\theta, x, y), \eta(\theta, x, y))
$$

the pullback condition $\Phi^{*} g=g$ is equivalent to the system

$$
\begin{aligned}
e^{2 A(\theta)} & =\left(\frac{\partial \tau}{\partial \theta}\right)^{2} e^{2 A(\tau)}+\left(\frac{\partial \xi}{\partial \theta}\right)^{2} e^{F+W(\tau)}+\left(\frac{\partial \eta}{\partial \theta}\right)^{2} e^{F-W(\tau)} \\
e^{F+W(\theta)} & =\left(\frac{\partial \tau}{\partial x}\right)^{2} e^{2 A(\tau)}+\left(\frac{\partial \xi}{\partial x}\right)^{2} e^{F+W(\tau)}+\left(\frac{\partial \eta}{\partial x}\right)^{2} e^{F-W(\tau)} \\
e^{F-W(\theta)} & =\left(\frac{\partial \tau}{\partial y}\right)^{2} e^{2 A(\tau)}+\left(\frac{\partial \xi}{\partial y}\right)^{2} e^{F+W(\tau)}+\left(\frac{\partial \eta}{\partial y}\right)^{2} e^{F-W(\tau)}
\end{aligned}
$$


Note that $s(\theta)$ is invertible, because $\partial s / \partial \theta=e^{A(\theta)}>0$, and define

$$
\begin{aligned}
& \tau(\theta, x, y)=s^{-1}\left(s(\theta)+s\left(\theta_{1}\right)-s\left(\theta_{0}\right)\right) \\
& \xi(\theta, x, y)=x_{1}+e^{-\frac{Z}{2}\left(s\left(\theta_{1}\right)-s\left(\theta_{0}\right)\right)}\left(x-x_{0}\right) \\
& \eta(\theta, x, y)=y_{1}+e^{\frac{Z}{2}\left(s\left(\theta_{1}\right)-s\left(\theta_{0}\right)\right)}\left(y-y_{0}\right) .
\end{aligned}
$$

Clearly, $\Phi: P_{0} \mapsto P_{1}$. Equation (2.7a) is satisfied, because

$$
\frac{\partial \tau}{\partial \theta}=\frac{\partial \theta}{\partial s}(\tau) \cdot \frac{\partial s}{\partial \theta}(\theta)=e^{-A(\tau)+A(\theta)} .
$$

To see that $(2.7 \mathrm{~b})$ is satisfied, let $\omega$ denote $W$ regarded as a linear function of arc length, so that $W(\theta)=\omega(s(\theta))$. Then we can write

$$
\begin{aligned}
\log \left(\left(\frac{\partial \xi}{\partial x}\right)^{2} e^{W(\tau)}\right) & =-Z \cdot\left(s\left(\theta_{1}\right)-s\left(\theta_{0}\right)\right)+\omega\left(s(\theta)+s\left(\theta_{1}\right)-s\left(\theta_{0}\right)\right) \\
& =\omega(s(\theta))=W(\theta) .
\end{aligned}
$$

Equation $(2.7 \mathrm{c})$ is verified in a similar fashion.

Remark 2.2. When studying a single locally homogeneous solv-Gowdy metric, one can always make $A$ constant in space by a reparameterization of $\mathcal{S}^{1}$; but it will not be convenient for us to do so.

If an arbitrary solv-Gowdy metric $g$ evolves by the Ricci flow

$$
\frac{\partial}{\partial t} g=-2 \mathrm{Rc}
$$

we shall abuse notation and allow the quantities introduced above to depend also on time. We find that $g$ remains a solv-Gowdy metric and that (2.8) is equivalent to the system

$$
\begin{aligned}
\frac{\partial}{\partial t} A & =\frac{1}{2} Z^{2} \\
\frac{\partial}{\partial t} W & =\frac{\partial}{\partial s} Z \\
\frac{\partial}{\partial t} F & =0,
\end{aligned}
$$

whose solution exists for all $t \geq 0$. It is most convenient to study $Z$ and recover $A$ and $W$ by integration. $Z$ evolves by

$$
\frac{\partial}{\partial t} Z=\frac{\partial^{2}}{\partial s^{2}} Z-\frac{1}{2} Z^{3}
$$


where the operator $\partial^{2} / \partial s^{2}$ plays the role of the Laplacian and evolves according to the commutator

$$
\left[\frac{\partial}{\partial t}, \frac{\partial}{\partial s}\right]=-\frac{1}{2} Z^{2} \frac{\partial}{\partial s} .
$$

For all $t \geq 0$, we identify $\mathcal{S}^{1}$ with the circle $x=0, y=0$ and denote its length by

$$
L(t) \doteqdot \int_{\mathcal{S}^{1}} d s=\int_{0}^{2 \pi} e^{A(\theta, t)} d \theta .
$$

Notice that (2.3) implies the important integral condition

$$
\int_{\mathcal{S}^{1}} Z d s=2 \log \lambda_{+}
$$

which is preserved by the flow.

If an evolving solv-Gowdy metric is locally homogeneous at $t=0$, it remains so under the Ricci flow. For such metrics, $Z$ is the function of time alone

$$
Z(t)=\frac{1}{\sqrt{t+1 / \zeta^{2}}},
$$

where $\zeta \doteqdot Z(0)$ is positive by (2.13). The sub-family of locally homogeneous solv-Gowdy metrics can thus be indexed by $(\alpha(\theta), \Omega, F)$, where

$$
\begin{aligned}
\alpha(\theta) & \doteqdot A(\theta, 0) \\
\Omega & \doteqdot W(0,0) .
\end{aligned}
$$

We now summarize the estimates we shall use from [1]. Let $g$ be a solution to the Ricci flow whose initial data $g(\cdot, 0)$ is a $C^{2}$ solv-Gowdy metric. Hamilton-Isenberg organize the proof of their main theorem into four steps. In Step 1, they show there is $C>0$ depending on $Z(\cdot, 0)$ such that for all $t>0$,

$$
|Z(\cdot, t)| \leq \frac{1}{\sqrt{t+C}}<\frac{1}{\sqrt{t}} .
$$

By Step 2, there is a time $T>0$ and constants $m \doteqdot Z_{\min }(T), M \doteqdot Z_{\max }(T)$ depending on $L(0), Z(\cdot, 0)$ and satisfying $0<m \leq M<1 / \sqrt{T}$ such that for all $t \geq T$,

$$
\frac{1}{\sqrt{t-T+1 / m^{2}}} \leq Z(\cdot, t) \leq \frac{1}{\sqrt{t-T+1 / M^{2}}} .
$$


By Step 1 again, there are $C, C^{\prime}>0$ depending on $L(0), Z(\cdot, 0)$ such that for all $t \geq T+1$,

$$
C \sqrt{t-T} \leq L(t) \leq C^{\prime} \sqrt{t-T} .
$$

By Step 4, there is $C>0$ depending on $L(0), Z(\cdot, 0)$ such that for all $t \geq T$,

$$
\left|\frac{\partial}{\partial s} Z(\cdot, t)\right| \leq \frac{C}{\left(1+m^{2}(t-T)\right)^{2}} .
$$

\section{Construction of approximating metrics.}

As a first step in proving the existence part (Theorem 4.1) of our main theorem, we find times $t_{\varepsilon}$ and construct locally homogeneous metrics $h_{\varepsilon}$ with the following properties: $h_{\varepsilon}$ is in a sense the average of $g$ at $t_{\varepsilon} ; h_{\varepsilon}$ remains $\varepsilon$-close to $g$ for all times $t \geq t_{\varepsilon}$; and most importantly, $h_{\varepsilon}$ exists for all $t \geq 0$.

Proposition 3.1. For any $\varepsilon>0$, there is a time $t_{\varepsilon}>0$ and a locally homogeneous solv-Gowdy metric $h_{\varepsilon}$ evolving by the Ricci flow for $0 \leq t<\infty$ such that

$$
\sup _{\mathcal{M}_{\Lambda}^{3} \times\left[t_{\varepsilon}, \infty\right)}\left|g-h_{\varepsilon}\right|_{h_{\varepsilon}}<\varepsilon .
$$

Before proving this, we collect some technical observations.

Lemma 3.2. For any $\varepsilon>0$, there is $t_{\varepsilon}>0$ such that $Z$ satisfies the pinching estimate

$$
Z_{\max }(t)-Z_{\min }(t) \leq \frac{\varepsilon}{L(t)},
$$

and the decay estimate

$$
\frac{1}{\sqrt{t-t_{\varepsilon}+1 / m_{\varepsilon}^{2}}} \leq Z(\cdot, t) \leq \frac{1}{\sqrt{t-t_{\varepsilon}+1 / M_{\varepsilon}^{2}}},
$$

for all $t \geq t_{\varepsilon}$, where $m_{\varepsilon}, M_{\varepsilon}$ are defined by

$$
0<m_{\varepsilon} \doteqdot Z_{\text {min }}\left(t_{\varepsilon}\right) \leq Z_{\text {max }}\left(t_{\varepsilon}\right) \doteqdot M_{\varepsilon}<\infty
$$

and satisfy

$$
m_{\varepsilon} \leq M_{\varepsilon} \leq m_{\varepsilon}+\varepsilon \quad \text { and } \quad M_{\varepsilon}^{2} \leq(1+\varepsilon) m_{\varepsilon}^{2} .
$$


Moreover, we can choose $t_{\varepsilon}$ so that

$$
\int_{t_{\varepsilon}}^{\infty}\left|\frac{\partial Z}{\partial s}\right| d t \leq \varepsilon
$$

Proof. Let $T, m, M$ be as in (2.17) and let $C$ be the constant in (2.19). Let $t_{*} \doteqdot \max \left\{T+C /\left(m^{4} \varepsilon\right), T+1\right\}$ and suppose $t \geq t_{*}$. Then (2.19) implies

$$
\int_{t_{*}}^{\infty}\left|\frac{\partial Z}{\partial s}\right| d t \leq \int_{0}^{\infty} \frac{C}{m^{4}\left(t+t_{*}-T\right)^{2}} d t=\frac{C}{m^{4}\left(t_{*}-T\right)} \leq \varepsilon
$$

and (2.18) implies there is $C^{\prime}>0$ such that

$$
L(t) \leq C^{\prime} \sqrt{t-T} .
$$

Hence for such times

$$
Z_{\max }(t)-Z_{\min }(t) \leq \int_{\mathcal{S}^{1}}\left|\frac{\partial Z}{\partial s}\right| d s \leq C C^{\prime} \frac{\sqrt{t-T}}{\left(1+m^{2}(t-T)\right)^{2}} .
$$

Choose $t_{\varepsilon} \geq t_{*}$ large enough that (3.1) holds for $t \geq t_{\varepsilon}$, and that (3.4) holds for $m_{\varepsilon}, M_{\varepsilon}$ defined by (3.3). This is possible, because

$$
\left(\frac{Z_{\max }(t)}{Z_{\min }(t)}\right)^{2} \leq \frac{t-T+1 / m^{2}}{t-T+1 / M^{2}} \leq 1+\frac{1}{m^{2}(t-T)} .
$$

Then since $\frac{\partial}{\partial t} Z=\frac{\partial^{2}}{\partial s^{2}} Z-\frac{1}{2} Z^{3}$, we observe that

$$
\frac{d}{d t} Z_{\min } \geq-\frac{1}{2} Z_{\min }^{3} \quad \text { and } \quad \frac{d}{d t} Z_{\max } \leq-\frac{1}{2} Z_{\max }^{3} .
$$

A routine use of the maximum principle (proved in [3]) now establishes (3.2) for all $t \geq t_{\varepsilon}$.

Remark 3.3. The proof shows that for $t \geq T+1$,

$$
Z_{\max }-Z_{\min }=O(t-T)^{-3 / 2},
$$

a result which also follows directly from (2.17). 
Lemma 3.4. Let $\varepsilon>0$ be given and let $t_{\varepsilon}, m_{\varepsilon}, M_{\varepsilon}$ be as in Lemma 3.2. Then there is a locally homogeneous solv-Gowdy metric

$$
h_{\varepsilon}=e^{2 A_{\varepsilon}} d \theta \otimes d \theta+e^{F_{\varepsilon}+W_{\varepsilon}} d x \otimes d x+e^{F_{\varepsilon}-W_{\varepsilon}} d y \otimes d y
$$

evolving by the Ricci flow for $0 \leq t<\infty$ so that for $t \geq t_{\varepsilon}$,

$$
\frac{1}{\sqrt{t-t_{\varepsilon}+1 / m_{\varepsilon}^{2}}} \leq Z_{\varepsilon}(t) \leq \frac{1}{\sqrt{t-t_{\varepsilon}+1 / M_{\varepsilon}^{2}}}
$$

where $Z_{\varepsilon}=\frac{\partial W_{\varepsilon}}{\partial s_{\varepsilon}}=e^{-A_{\varepsilon} \frac{\partial W_{\varepsilon}}{\partial \theta}}$. Moreover, $h_{\varepsilon}$ is constructed so that for all $\theta \in \mathcal{S}^{1}, A_{\varepsilon}\left(\theta, t_{\varepsilon}\right)=A\left(\theta, t_{\varepsilon}\right)$ and $\left|W\left(\theta, t_{\varepsilon}\right)-W_{\varepsilon}\left(\theta, t_{\varepsilon}\right)\right| \leq \varepsilon$.

Proof. Define

$$
Z_{\varepsilon}(t) \doteqdot \frac{1}{\sqrt{t+\left(1 / \zeta_{\varepsilon}^{2}-t_{\varepsilon}\right)}}
$$

where

$$
\zeta_{\varepsilon} \doteqdot \int_{\mathcal{S}^{1}} Z d s / \int_{\mathcal{S}^{1}} d s
$$

with the RHS evaluated at $t_{\varepsilon}$. Observe that $Z_{\varepsilon}$ is well defined for all $t \geq 0$, because $|Z(t)|<1 / \sqrt{t}$ by $(2.16)$, whence

$$
1 / \zeta_{\varepsilon}^{2}-t_{\varepsilon} \geq 1 / Z_{\max }^{2}\left(t_{\varepsilon}\right)-t_{\varepsilon}>0
$$

Now recall that locally homogeneous solv-Gowdy metrics form a 3-parameter family and define

$$
\begin{aligned}
\alpha_{\varepsilon}(\theta) & \doteqdot A\left(\theta, t_{\varepsilon}\right)-\frac{1}{2} \int_{0}^{t_{\varepsilon}} Z_{\varepsilon}^{2} d t \\
\Omega_{\varepsilon} & \doteqdot W\left(0, t_{\varepsilon}\right) \\
F_{\varepsilon} & \doteqdot F .
\end{aligned}
$$

Notice that $h_{\varepsilon}$ is well defined; indeed, the identities

$$
2 \log \lambda_{+}=\int_{\mathcal{S}^{1}} Z d s=\zeta_{\varepsilon} \int_{\mathcal{S}^{1}} d s=\int_{\mathcal{S}^{1}} \zeta_{\varepsilon} e^{A_{\varepsilon}} d \theta=\int_{\mathcal{S}^{1}} Z_{\varepsilon} d s_{\varepsilon}
$$

show that the integral condition (2.13) is satisfied at $t_{\varepsilon}$, hence for all time. 
The first assertion of the lemma is verified by the elementary observation

$$
m_{\varepsilon}=Z_{\min }\left(t_{\varepsilon}\right) \leq \zeta_{\varepsilon} \leq Z_{\max }\left(t_{\varepsilon}\right)=M_{\varepsilon}
$$

which follows from (3.6). The second assertion is trivial; to prove the third, simply notice that

$$
\left|W\left(\theta, t_{\varepsilon}\right)-W_{\varepsilon}\left(\theta, t_{\varepsilon}\right)\right| \leq \int_{\mathcal{S}^{1}}\left|Z-\zeta_{\varepsilon}\right| d s \leq\left(Z_{\max }-Z_{\min }\right)\left(t_{\varepsilon}\right) \cdot L\left(t_{\varepsilon}\right) \leq \varepsilon .
$$

Proof of Proposition 3.1. Without loss of generality, assume $0<\varepsilon \leq 1 / 6$.

Let $t \geq t_{\varepsilon}$ and observe that

$$
\begin{aligned}
\left|\left(A-A_{\varepsilon}\right)(\theta, t)\right| & =\frac{1}{2}\left|\int_{t_{\varepsilon}}^{t}\left(Z^{2}-Z_{\varepsilon}^{2}\right)(\theta, \tau) d \tau\right| \\
& \leq \frac{1}{2} \int_{t_{\varepsilon}}^{t}\left(\frac{1}{\tau-t_{\varepsilon}+1 / M_{\varepsilon}^{2}}-\frac{1}{\tau-t_{\varepsilon}+1 / m_{\varepsilon}^{2}}\right) d \tau \\
& =\log \sqrt{\frac{1+M_{\varepsilon}^{2}\left(t-t_{\varepsilon}\right)}{1+m_{\varepsilon}^{2}\left(t-t_{\varepsilon}\right)}} .
\end{aligned}
$$

Then since $\left|e^{u}-1\right| \leq e^{U}-1$ when $|u| \leq U$, we have

$$
\left|\left(e^{2 A}-e^{2 A_{\varepsilon}}\right)(\theta, t)\right|=e^{2 A_{\varepsilon}}\left|e^{2\left(A-A_{\varepsilon}\right)}-1\right| \leq e^{2 A_{\varepsilon}} \frac{M_{\varepsilon}^{2}-m_{\varepsilon}^{2}}{m_{\varepsilon}^{2}}
$$

and hence

$$
\left(\left(h_{\varepsilon}\right)^{\theta \theta}\right)^{2}\left(g_{\theta \theta}-\left(h_{\varepsilon}\right)_{\theta \theta}\right)^{2} \leq \varepsilon^{2} .
$$

Because $W_{\varepsilon}$ is constant in time, we have

$$
\begin{aligned}
\left|\left(W-W_{\varepsilon}\right)(\theta, t)\right| & \leq\left|W(\theta, t)-W\left(\theta, t_{\varepsilon}\right)\right|+\left|W\left(\theta, t_{\varepsilon}\right)-W_{\varepsilon}\left(\theta, t_{\varepsilon}\right)\right| \\
& \leq\left|\int_{t_{\varepsilon}}^{t} \frac{\partial Z}{\partial s} d \tau\right|+\varepsilon \\
& \leq 2 \varepsilon
\end{aligned}
$$

whence substituting $\delta=2 \varepsilon \leq 1 / 3$ in the crude estimate $e^{\delta} \leq 1+\delta+\frac{e}{2} \delta^{2}$ (which holds for $0 \leq \delta \leq 1$ ) gives

$$
\left|\left(e^{F+W}-e^{F_{\varepsilon}+W_{\varepsilon}}\right)(\theta, t)\right|=e^{F_{\varepsilon}+W_{\varepsilon}}\left|e^{\left(W-W_{\varepsilon}\right)}-1\right| \leq 3 \varepsilon e^{F_{\varepsilon}+W_{\varepsilon}}
$$


and thus

$$
\left(\left(h_{\varepsilon}\right)^{x x}\right)^{2}\left(g_{x x}-\left(h_{\varepsilon}\right)_{x x}\right)^{2} \leq 9 \varepsilon^{2} .
$$

The estimate for $\left(\left(h_{\varepsilon}\right)^{y y}\right)^{2}\left(g_{y y}-\left(h_{\varepsilon}\right)_{y y}\right)^{2}$ is entirely analogous. We have shown that

$$
\left|g-h_{\varepsilon}\right|_{h_{\varepsilon}}^{2}=\left(h_{\varepsilon}\right)^{a c}\left(h_{\varepsilon}\right)^{b d}\left(g_{a b}-\left(h_{\varepsilon}\right)_{a b}\right)\left(g_{c d}-\left(h_{\varepsilon}\right)_{c d}\right) \leq 19 \varepsilon^{2}
$$

for $t \geq t_{\varepsilon}$, which is clearly equivalent to the desired result.

\section{Existence.}

We have seen that for any $\varepsilon>0$, there is a natural choice $h_{\varepsilon}$ of locally homogeneous metric approximating $g$ for times $t \geq t_{\varepsilon}$. In view of our nonuniqueness result (Theorem 5.1), it is remarkable that these choices are close enough to one another that we can prove the existence of a locally homogeneous metric in $[g]$.

Theorem 4.1. There is a locally homogeneous solv-Gowdy metric $h_{\mathrm{co}}$ evolving by the Ricci flow for $0 \leq t<\infty$ such that for any $\varepsilon>0$ there is a time $t_{\varepsilon}>0$ with

$$
\sup _{\mathcal{M}_{\Lambda}^{3} \times\left[t_{\varepsilon}, \infty\right)}\left|g-h_{\infty}\right|_{h_{\infty}}<\varepsilon .
$$

Again, we first obtain some preliminary results.

Lemma 4.2. Let $\left\{\varepsilon_{j}\right\}$ be a sequence with $\varepsilon_{j} \searrow 0$. For each $j$, let $h_{j}$ denote the metric $h_{\varepsilon_{j}}$ given by Proposition 3.1. Then there is a subsequence $j_{k}$ and a locally homogeneous metric $h_{\infty}$ with data $\left(\alpha_{\infty}(\theta), \Omega_{\infty}, F_{\infty}\right)$ such that

$$
\left(\alpha_{j_{k}}(\theta), \Omega_{j_{k}}, F_{j_{k}}\right) \rightarrow\left(\alpha_{\infty}(\theta), \Omega_{\infty}, F_{\infty}\right)
$$

uniformly in $\theta$. (Here, and throughout the proof, a subscript such as $j$ denotes quantities corresponding to the metric $h_{j} \equiv h_{\varepsilon_{j}}$.)

Proof. The argument is constructed from four claims, as follows: Claim 4.3 bounds $\frac{\partial}{\partial \theta} A\left(\cdot, t_{j}\right)$, hence $\frac{\partial}{\partial \theta} A_{j}\left(\cdot, t_{j}\right)$ by construction, hence $\frac{\partial}{\partial \theta} A_{j}(\cdot, 0)$ by (4.1) and the local homogeneity of $h_{j}$. Combining this with Claim 4.4 proves $\left\{A_{j}(\cdot, 0)\right\}$ is bounded and equicontinuous. Since Claim 4.5 bounds 
$\frac{\partial}{\partial s_{j}} W_{j}(\cdot, 0)$, this lets us bound $\frac{\partial}{\partial \theta} W_{j}(\cdot, 0)$. Combining this with Claim 4.6 then proves $\left\{W_{j}(\cdot, 0)\right\}$ is bounded and equicontinuous. Because $F_{j} \equiv F$ by construction, this lets us extract a subsequence of the $h_{j}$ whose initial data converge uniformly to the data of a locally homogeneous metric $h_{\infty}$ existing for all $t \geq 0$.

Notice that if $j<k$, we may (and shall) assume $t_{j} \leq t_{k}$.

Claim 4.3. There is $C<\infty$ such that

$$
\sup _{\mathcal{M}_{\Lambda}^{3} \times[T, \infty)}\left|\frac{\partial A}{\partial \theta}\right|<C .
$$

Compute

$$
\frac{\partial}{\partial t}\left(\frac{\partial A}{\partial \theta}\right)=\frac{\partial}{\partial \theta}\left(\frac{1}{2} Z^{2}\right)=e^{A} Z \frac{\partial Z}{\partial s}
$$

Since by $(2.17)$,

$$
\frac{\partial}{\partial t} A \leq \frac{1}{2} \cdot \frac{1}{t-T+1 / M^{2}}
$$

for $t \geq T$, there is $C^{\prime}>0$ such that

$$
A(\cdot, t) \leq \log C^{\prime}+\log \sqrt{t-T+1 / M^{2}}
$$

for $t \geq T$. Then by (2.19), we have

$$
\begin{aligned}
\left|\frac{\partial}{\partial t}\left(\frac{\partial A}{\partial \theta}\right)\right| & \leq C^{\prime} \sqrt{t-T+1 / M^{2}} \frac{1}{\sqrt{t-T+1 / M^{2}}} \cdot \frac{C^{\prime \prime}}{\left(1+m^{2}(t-T)\right)^{2}} \\
& \leq \frac{C^{\prime} C^{\prime \prime}}{1+m^{4}(t-T)^{2}}
\end{aligned}
$$

for all $t \geq T$. Since there is $B>0$ depending only on the initial data such that $-B \leq \partial A / \partial \theta \leq B$ at $t=T$, the claim follows.

Claim 4.4. The sequence $\left\{\alpha_{j}(\theta)\right\}$ is bounded for each $\theta \in S^{1}$.

Let $\theta \in \mathcal{S}^{1}$ be arbitrary. For $j<k$, consider

$$
\begin{aligned}
\alpha_{j}(\theta)-\alpha_{k}(\theta) & =A\left(\theta, t_{j}\right)-\frac{1}{2} \int_{0}^{t_{j}} Z_{j}^{2} d t-A\left(\theta, t_{k}\right)+\frac{1}{2} \int_{0}^{t_{k}} Z_{k}^{2} d t \\
& =\frac{1}{2} \int_{t_{j}}^{t_{k}}\left(Z_{k}^{2}-Z^{2}\right) d t+\frac{1}{2} \int_{0}^{t_{j}}\left(Z_{k}^{2}-Z_{j}^{2}\right) d t .
\end{aligned}
$$


Since $1 / \zeta_{k}^{2}-t_{k} \geq 1 / M_{j}^{2}-t_{j}$, we obtain a familiar estimate for the first integral:

$$
\frac{1}{2}\left|\int_{t_{j}}^{t_{k}}\left(Z_{k}^{2}-Z^{2}\right) d t\right| \leq \log \sqrt{\frac{1+M_{j}^{2}\left(t_{k}-t_{j}\right)}{1+m_{j}^{2}\left(t_{k}-t_{j}\right)}} \leq \log \sqrt{1+\varepsilon_{j}} .
$$

Write the second integral as

$$
\begin{aligned}
\frac{1}{2} \int_{0}^{t_{j}}\left(Z_{k}^{2}-Z_{j}^{2}\right) d t & =\log \sqrt{\frac{1 / \zeta_{j}^{2}-t_{j}}{1 / \zeta_{k}^{2}-t_{k}}}+\log \sqrt{\frac{t_{j}+\left(1 / \zeta_{k}^{2}-t_{k}\right)}{1 / \zeta_{j}^{2}}} \\
& =\log \sqrt{P_{j k}}
\end{aligned}
$$

where

$$
P_{j k} \doteqdot\left(1-\zeta_{j}^{2} t_{j}\right)\left(1+\frac{t_{j}}{1 / \zeta_{k}^{2}-t_{k}}\right)>0
$$

Since

$$
\frac{1 / M^{2}-T}{t_{j}+1 / M^{2}-T} \leq 1-\zeta_{j}^{2} t_{j} \leq \frac{1 / m^{2}-T}{t_{j}+1 / m^{2}-T}
$$

and

$$
\frac{t_{j}+1 / m^{2}-T}{1 / m^{2}-T} \leq 1+\frac{t_{j}}{1 / \zeta_{k}^{2}-t_{k}} \leq \frac{t_{j}+1 / M^{2}-T}{1 / M^{2}-T}
$$

we conclude that

$$
\frac{1 / M^{2}-T}{1 / m^{2}-T} \leq P_{j k} \leq \frac{1 / m^{2}-T}{1 / M^{2}-T}
$$

Claim 4.5. There are $0<Z_{*} \leq Z^{*}<\infty$ such that $Z_{j}(0) \in\left[Z_{*}, Z^{*}\right]$ for all $j$.

Note how

$$
1 / Z_{j}^{2}(0)=1 / \zeta_{j}^{2}-t_{j} \geq 1 / Z_{\max }^{2}\left(t_{j}\right)-t_{j} \geq 1 / M^{2}-T>0
$$

by (2.16) and (2.17), and similarly

$$
1 / Z_{j}^{2}(0)=1 / \zeta_{j}^{2}-t_{j} \leq 1 / Z_{\min }^{2}\left(t_{j}\right)-t_{j} \leq 1 / m^{2}-T<\infty .
$$

Claim 4.6. There are $\Omega_{*} \leq \Omega^{*}$ such that $\Omega_{j} \in\left[\Omega_{*}, \Omega^{*}\right]$ for all $j$. 
Suppose $j<k$. Then since $\Omega_{j} \doteqdot W\left(0, t_{j}\right)$, we have

$$
\left|\Omega_{k}-\Omega_{j}\right|=\left|W\left(0, t_{k}\right)-W\left(0, t_{j}\right)\right| \leq \int_{t_{j}}^{t_{k}}\left|\frac{\partial W}{\partial t}\right| d t=\int_{t_{j}}^{t_{k}}\left|\frac{\partial Z}{\partial s}\right| d t \leq \varepsilon_{j} .
$$

Lemma 4.7. If $h_{\infty}$ is a locally homogeneous metric with data

$$
\left(\alpha_{\infty}(\theta), \Omega_{\infty}, F\right)
$$

and $\left\{h_{j}\right\}$ is a sequence of locally homogeneous metrics with data

$$
\left(\alpha_{j}(\theta), \Omega_{j}, F\right)
$$

converging to $\left(\alpha_{\infty}(\theta), \Omega_{\infty}, F\right)$ uniformly in $\theta$, then for any $\varepsilon>0$ there is $J_{\varepsilon}$ such that for each $j \geq J_{\varepsilon}$

$$
\sup _{\mathcal{M}_{\Lambda}^{3} \times[0, \infty)}\left|h_{j}-h_{\infty}\right|_{h_{\infty}}<\varepsilon .
$$

Proof. The integral condition

$$
\int_{\mathcal{S}^{1}} Z_{\infty}(0) e^{\alpha_{\infty}(\theta)} d \theta=2 \log \lambda_{+}=\int_{\mathcal{S}^{1}} Z_{j}(0) e^{\alpha_{j}(\theta)} d \theta
$$

shows that $Z_{j}(0) \rightarrow Z_{\infty}(0)$. For $\delta>0$ to be determined, choose $J_{\varepsilon}$ large enough that

$$
\sup _{\theta \in \mathcal{S}^{1}}\left|\alpha_{\infty}(\theta)-\alpha_{j}(\theta)\right| \leq \delta \quad \text { and } \quad\left|\frac{Z_{\infty}^{2}(0)}{Z_{j}^{2}(0)}-1\right| \leq \delta
$$

for all $j \geq J_{\varepsilon}$, and consider

$$
\left(A_{\infty}-A_{j}\right)(\theta, t)=\left(\alpha_{\infty}-\alpha_{j}\right)(\theta)+\frac{1}{2} \int_{0}^{t}\left(Z_{\infty}^{2}-Z_{j}^{2}\right) d t .
$$

For any $\lambda, \mu>0$ we have the now-familiar inequality

$$
\log \left(1-\frac{|\mu-\lambda|}{\lambda}\right) \leq \int_{0}^{t}\left(\frac{1}{t+\lambda}-\frac{1}{t+\mu}\right) d t \leq \log \left(1+\frac{|\mu-\lambda|}{\lambda}\right) .
$$

Since

$$
\frac{1}{2} \int_{0}^{t}\left(Z_{\infty}^{2}-Z_{j}^{2}\right) d t=\frac{1}{2} \int_{0}^{t}\left(\frac{1}{t+1 / Z_{\infty}^{2}(0)}-\frac{1}{t+1 / Z_{j}^{2}(0)}\right) d t
$$


and

$$
\frac{\left|1 / Z_{j}^{2}(0)-1 / Z_{\infty}^{2}(0)\right|}{1 / Z_{\infty}^{2}(0)} \leq \delta
$$

we get our first estimate:

$$
\left|\left(A_{\infty}-A_{j}\right)(\theta, t)\right| \leq \delta+\log \sqrt{1+\delta} .
$$

Next observe that when $0<\delta \leq \log 2$ we have $e^{\delta} \leq 1+2 \delta$ and thus obtain our second estimate:

$$
\begin{aligned}
\left|\left(W_{\infty}-W_{j}\right)(\theta, t)\right| & =\left|W_{\infty}(\theta, 0)-W_{j}(\theta, 0)\right| \\
& =\left|\int_{0}^{\theta} Z_{\infty}(0) \cdot e^{\alpha_{\infty}(u)} d u-\int_{0}^{\theta} Z_{j}(0) \cdot e^{\alpha_{j}(u)} d u\right| \\
& \leq \int_{0}^{\theta} Z_{\infty}(0) \cdot e^{\alpha_{\infty}(u)}\left|1-e^{\alpha_{j}(u)-\alpha_{\infty}(u)}\right| d u \\
& +\int_{0}^{\theta} Z_{j}(0) \cdot e^{\alpha_{j}(u)}\left|\frac{Z_{\infty}(0)}{Z_{j}(0)}-1\right| d u \\
& \leq 3 \delta\left(2 \log \lambda_{+}\right) .
\end{aligned}
$$

As in the proof of Theorem 3.1, it follows that we can make $\left|h_{\infty}-h_{j}\right|_{h_{\infty}}$ as small as desired by choosing $\delta=\delta(\varepsilon)$ appropriately.

Proof of Theorem 4.1. Note that $\left|g-h_{\infty}\right|_{h_{\infty}}$ will be small if both $\left|g-h_{j}\right|_{h_{j}}$ and $\left|h_{j}-h_{\infty}\right|_{h_{\infty}}$ are. So take the subsequence of metrics $h_{j_{k}}$ and times $t_{j_{k}}$ given by Lemma 4.2 and pass to a further subsequence according to Lemma 4.7.

\section{Uniqueness.}

Distinct locally homogeneous solv-Gowdy metrics belong to the same equivalence class if and only if they differ merely by a dilation of arc length. In that case, we shall see that they approach one another at the rate $C / t$, where the constant depends on the initial difference in length of the base circle.

Theorem 5.1. Let $h$ and $h_{*}$ be locally homogeneous metrics corresponding to the data $(\alpha(\theta), \Omega, F)$ and $\left(\alpha_{*}(\theta), \Omega_{*}, F_{*}\right)$ respectively. If for some 
constant $\ell$ we have $\alpha_{*} \equiv \alpha+\ell$ and $\Omega_{*}=\Omega$ and $F_{*}=F$, then $h$ and $h_{*}$ quasi-converge with

$$
\left|h_{*}-h\right|_{h}=O\left(\frac{1}{t}\right) \text {. }
$$

In all other cases, there are $\delta>0$ and $\theta \in \mathcal{S}^{1}$ such that

$$
\left|h_{*}-h\right|_{h}(\theta, t) \geq \delta
$$

for all $t>0$, so $h$ and $h_{*}$ do not quasi-converge.

Proof. We consider three cases.

Case 5.2. $\alpha_{*} \equiv \alpha+\ell, \Omega_{*}=\Omega, F_{*}=F$.

Writing

$$
Z(t)=\frac{1}{\sqrt{t+1 / \zeta^{2}}} \quad \text { and } \quad Z_{*}(t)=\frac{1}{\sqrt{t+1 / \zeta_{*}^{2}}}
$$

we observe that $\ell=\log \left(\zeta / \zeta_{*}\right)$, since by the integral condition (2.13) we have

$$
\frac{\zeta}{\zeta_{*}}=\frac{\int_{\mathcal{S}^{1}} e^{\alpha_{*}(\theta)} d \theta}{\int_{\mathcal{S}^{1}} e^{\alpha(\theta)} d \theta}=e^{\ell}
$$

It follows that the function

$$
\omega(\theta) \doteqdot \int_{0}^{\theta}\left(\zeta_{*} e^{\alpha_{*}(u)}-\zeta e^{\alpha(u)}\right) d u
$$

is identically zero. So for all $\theta \in \mathcal{S}^{1}$ and $t \geq 0$ we have

$$
\left(W_{*}-W\right)(\theta, t)=\left(W_{*}-W\right)(\theta, 0)=\Omega_{*}-\Omega+\omega(\theta)=0 .
$$

Now notice that

$$
\left(A_{*}-A\right)(\theta, t)=\left(\alpha_{*}-\alpha\right)(\theta)+\frac{1}{2} \int_{0}^{t}\left(Z_{*}^{2}(\tau)-Z^{2}(\tau)\right) d \tau=\ell+\phi(t),
$$

where

$$
\phi(t) \doteqdot \frac{1}{2} \log \frac{1+\zeta_{*}^{2} t}{1+\zeta^{2} t}
$$


It is clear by (5.1) that $A_{*}-A \rightarrow 0$ uniformly in $\theta$ as $t \rightarrow \infty$. In fact, this identifies the critical rate at which distinct locally homogeneous metrics $h, h_{*}$ approach each other, because

$$
\left(e^{2 A_{*}}-e^{2 A}\right)(\theta, t)=e^{2 A(\theta, t)}\left(e^{2(\ell+\phi(t))}-1\right)
$$

and hence

$$
\left|h_{*}-h\right|_{h}=\left|h^{\theta \theta}\left(h_{*}-h\right)_{\theta \theta}\right|=\left|e^{2(\ell+\phi(t))}-1\right|=\frac{\left|1 / \zeta_{*}^{2}-1 / \zeta^{2}\right|}{t+1 / \zeta^{2}} .
$$

Case 5.3. $\alpha_{*} \equiv \alpha+\ell, \Omega_{*}=\Omega, F_{*} \neq F$.

Notice that $W_{*}-W \equiv 0$ and $A_{*}-A \rightarrow 0$ as above. Without loss of generality, suppose $F_{*}-F=\delta>0$. Then for all $\theta \in \mathcal{S}^{1}$ and $t \geq 0$ we have

$$
e^{F_{*}+W_{*}}-e^{F+W}=e^{F+W}\left(e^{F_{*}-F}-1\right)>\delta e^{F+W}
$$

and hence

$$
\left|h_{*}-h\right|_{h} \geq\left|h^{x x}\left(h_{*}-h\right)_{x x}\right|>\delta>0 .
$$

Case 5.4. Either $\alpha_{*} \not \equiv \alpha+\ell$ or $\Omega_{*} \neq \Omega$.

Observe that we can always find $\theta$ with

$$
\left(W_{*}-W\right)(\theta, 0)=\Omega_{*}-\Omega+\omega(\theta) \neq 0,
$$

since $\omega$ cannot be identically zero if $\alpha_{*} \not \equiv \alpha+\ell$. Without loss of generality, assume $\left(W_{*}-W\right)(\theta, 0)=\delta>0$. Then if $F_{*} \geq F$, we have

$$
e^{F_{*}+W_{*}(\theta, t)}-e^{F+W(\theta, t)}=e^{F+W(\theta, t)}\left(e^{F_{*}-F} e^{\delta}-1\right) \geq e^{F+W(\theta, t)}\left(e^{\delta}-1\right)
$$

for all $t \geq 0$ and hence

$$
\left|h_{*}-h\right|_{h}(\theta, t) \geq\left|h^{x x}\left(h_{*}-h\right)_{x x}\right|(\theta, t)>\delta>0 .
$$

On the other hand, if $F \geq F_{*}$ we obtain

$$
e^{F_{*}-W_{*}(\theta, t)}-e^{F-W(\theta, t)}=e^{F-W(\theta, t)}\left(e^{F_{*}-F} e^{-\delta}-1\right) \leq e^{F-W(\theta, t)}\left(e^{-\delta}-1\right)
$$

for all $t \geq 0$ and thus

$$
\left|h_{*}-h\right|_{h}(\theta, t) \geq\left|h^{y y}\left(h_{*}-h\right)_{y y}\right|(\theta, t)>\frac{\delta}{1+\delta}>0 .
$$




\section{References.}

[1] R. Hamilton and J. Isenberg, Quasi-convergence of Ricci flow for a class of metrics, Comm. Anal. Geom. 1:4 (1993), 543-559.

[2] M. Carfora, J. Isenberg, and M. Jackson, Convergence of the Ricci flow for metrics with indefinite curvature, J. Differential Geom. 31 (1990), 249-263.

[3] R. Hamilton, Four-manifolds with positive curvature operator, J. Differential Geom. 24 (1986), 153-179.

UNIVERSITY OF WISCONSIN

MILWAUKEE WI

USA 